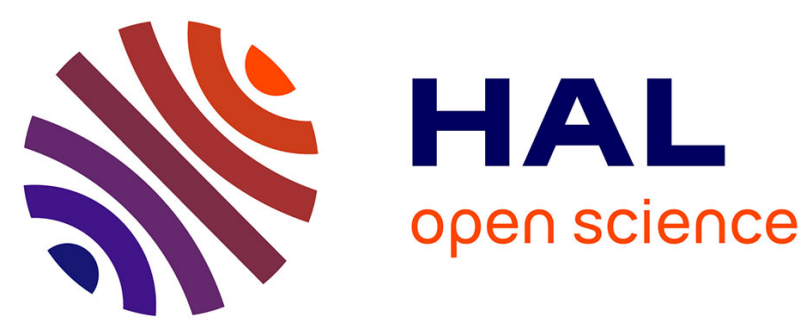

\title{
Perturbation model for the control of the spectral properties of high contrast gratings
}

Cédric Blanchard, Cécile Jamois, Pierre Viktorovitch, Christian Grillet, Jean-Louis Leclercq, Taha Benyattou, Xavier Letartre

\section{To cite this version:}

Cédric Blanchard, Cécile Jamois, Pierre Viktorovitch, Christian Grillet, Jean-Louis Leclercq, et al.. Perturbation model for the control of the spectral properties of high contrast gratings. SPIE Photonics West 2015, Feb 2015, San Francisco, United States. 10.1117/12.2076595 . hal-01489309

\section{HAL Id: hal-01489309 https://hal.science/hal-01489309}

Submitted on 29 Jun 2021

HAL is a multi-disciplinary open access archive for the deposit and dissemination of scientific research documents, whether they are published or not. The documents may come from teaching and research institutions in France or abroad, or from public or private research centers.
L'archive ouverte pluridisciplinaire HAL, est destinée au dépôt et à la diffusion de documents scientifiques de niveau recherche, publiés ou non, émanant des établissements d'enseignement et de recherche français ou étrangers, des laboratoires publics ou privés.

\section{(c)(1)}

Distributed under a Creative Commons Attribution| 4.0 International License 


\title{
Perturbation model for the control of the spectral properties of high contrast gratings
}

\author{
${ }^{a}$ Cédric Blanchard, ${ }^{b}$ Cécile Jamois, ${ }^{a}$ Pierre Viktorovitch, ${ }^{a}$ Christian Grillet, ${ }^{a}$ Jean-Louis \\ Leclercq, ${ }^{b}$ Taha Benyattou, and ${ }^{a}$ Xavier Letartre. \\ ${ }^{a}$ University of Lyon. Institut des Nanotechnologies de Lyon. CNRS UMR5270. École Centrale \\ de Lyon, 36 Avenue Guy de Collongue, 69134 Ecully, France. \\ ${ }^{b}$ University of Lyon. Institut des Nanotechnologies de Lyon. CNRS UMR5270. Institut \\ National des Sciences Appliquées, 7 avenue Jean Capelle, 69621 Villeurbanne, France.
}

\begin{abstract}
The comprehension and manipulation of the spectral characteristics of photonic structures is of great interest for a vast bunch of applications, in particular for energy efficiency. In this paper we focus on a perturbation model capable of providing insight and control on the resonances that are supported by high index contrast gratings.
\end{abstract}

Keywords: Photonic Crystal, high index contrast membrane, perturbation model.

\section{INTRODUCTION}

Energy conservation is a primary objective for countries with high amounts of energy consumption. This arises from fears surrounding the exhaustion of natural resources across the globe (i.e. fossil fuels); global warming caused by the production of greenhouse gases; or the increase of the energy cost, which is highly detrimental to economic activity. Not to mention, since access to energy is essential for modern economies, there are also geopolitical reasons towards reducing energy consumption. The goal of reducing the energy consumption necessitates efficient energy use; less energy for a constant service. As an example, building insulation or compact fluorescent lights contribute to energy efficiency with no disruption to a person's lifestyle.

We are currently witnessing the emergence of a new frontier in scientific research through the prominence that is given to the development of new concepts and technologies enabling a drastic reduction in energy consumption. For example if we focused on data centers, which exhibit an extraordinary high energy demand, one of the primary reasons contributing to high power and cost factors is cooling. In this context, the passive radiating cooling strategy developed in the team of S. Fan may have a significant impact. ${ }^{1,2}$ In this approach a multilayered material is engineered so that it selectively emits in the atmospheric transparency window and acts as a highly efficient mirror for visible light. In this way the infrared light, containing heat, is radiated into the cold darkness of space while sunlight is reflected in order to prevent incoming heat. The cornerstone of this nanophotonic approach is the precise tailoring of the dispersion characteristics of the structure.

Together with energy efficiency, renewable energy is the other big constitutive block of any sustainable energy policy. A team at MIT recently reported on a full solar thermophotovoltaic device able to reach significantly high experimental efficiencies. ${ }^{3}$ It includes a black absorber which, when exposed to sunlight, heats up and eventually radiates towards the photovoltaic cell. Efficient solar energy conversion is then achieved through the control of light that reaches the photovoltaic element. Concretely, a photonic crystal structure allows the spectral matching to occur between the thermal radiation and the absorption of the employed semiconductor.

The Stanford's radiative cooling and MIT's thermophotovoltaic devices are both based on the tight control of the spectral properties by means of a nanophotonic system. The main purpose of this article is to present with great details a perturbative model that affords insight into the spectral properties of high contrast gratings - also called Photonic crystal membranes (PCMs) - and, in particular, into the mechanism that allows the obtention of resonances endowed with extremely high quality factors.

Further author information: Send correspondence to Cédric Blanchard. cjfblanchard@gmail.com. 
The model is based on a semi-analytical approach that was introduced by Kazarinov and Henry (KH) to discuss the effect of radiation losses on a distributed feedback (DFB) laser. ${ }^{4}$ We show here how to extend KH's work to the study of PCMs. ${ }^{5}$ We point out that the dispersion relation arising from the model is capable of accounting for both the bandgap and radiation losses suffered by the PCM. Since we are dealing with a perturbative approach, the information provided by the model should only be valid for small index contrast gratings. But in practice it proves robust for high index contrast gratings as well.

\section{PERTURBATION MODEL}

\subsection{Kazarinov and Henry's model}

The purpose of this section is to provide the equations governing the model that was proposed by KH in 1985 . More than a simple reminder, the following lines are important to understand the essence of the perturbative approach. The subsequent developments of this article are based on two parameters $h_{1}$ and $h_{2}$, given in Eq.(20). The relationship between them is furnished in Eq.(21), it establishes the coupling between the structure and the guided and radiated waves.

We consider a two-dimensional PCM with thickness $d$ and that is periodic along the $z$-direction. Accordingly the permittivity $\epsilon(x, z)$ can be expanded into a Fourier series that takes the following form:

$$
\epsilon(x, z)=\nu(x)+\epsilon_{p c m}(x, z)=\nu(x)-\Delta \epsilon \sum_{m \neq 0} \xi_{m}(x) \mathrm{e}^{-i K m z} .
$$

In this expression $\nu(x)$ is the permittivity outside the corrugation and $\epsilon_{p c m}(x, z)$ is a function that is non-zero only into the corrugated region. $\Delta \epsilon$ is the index contrast between the two constitutive materials of the PCM, and $\xi_{m}$ are the Fourier's coefficients of the expansion,

$$
\xi_{m}=\frac{\sin m \pi F}{m \pi}
$$

$F$ being the duty cycle of the PCM.

The field inside the membrane is given by the wave equation

$$
\nabla^{2} E+k^{2} E=0
$$

with $k=n \omega / c$ ( $n$ is the refractive index). From this point onward, the approach is going perturbative; we assume that when a plane wave is normally incident on the PCM, the electric field in the PCM can be written as:

$$
E(x, z)=\left[A(z) \mathrm{e}^{i K z}+B(z) \mathrm{e}^{-i K z}\right] \Phi(x)+\Delta E(x, z) .
$$

The first term of this expression is reminiscent of the field into a homogeneous waveguide. Note, however, the z-dependance of the $A$ and $B$ coefficients. It is important to understand that the function $\Phi(x)$ represents the profile of the guided wave into the bare membrane (with no corrugation) even if we are dealing with a PCM. The wave vector of the grating, $K=2 \pi / \Lambda$, comes on its own in the argument of the exponentials because the incoming wave is perpendicular to the guide (the propagation constant $\beta=k_{z}+K$ is reduced since $k_{z}=0$ ). The period of the PCM is denoted $\Lambda$. The radiating waves, due to the corrugation of the waveguide (corrugation which can be seen as a perturbation), are contained into the term $\Delta E(x, z)$.

By introducing Eqs.(1,4) into Eq.(3) and by solely considering $m=-2,-1,1,2$ in the summation, we obtain an expression of the form

$$
\alpha_{-1} \mathrm{e}^{-i K z}+\alpha_{0}+\alpha_{+1} \mathrm{e}^{i K z}=0,
$$

with

$$
\begin{aligned}
& \alpha_{+1}=-\frac{\Delta \epsilon \omega^{2} \xi_{-1} \Delta E}{c^{2}}-K^{2} A \Phi-\frac{\Delta \epsilon \omega^{2} \xi_{-2} B \Phi}{c^{2}}+\frac{\omega^{2} \nu A \Phi}{c^{2}}+2 i K A^{\prime} \Phi(x)+A^{\prime \prime} \Phi+A \Phi^{\prime \prime} \\
& \alpha_{-1}=-\frac{\Delta \epsilon \omega^{2} \xi_{+1} \Delta E}{c^{2}}-K^{2} B \Phi-\frac{\Delta \epsilon \omega^{2} \xi_{+2} A \Phi}{c^{2}}+\frac{\omega^{2} \nu B \Phi}{c^{2}}-2 i K B^{\prime} \Phi(x)+B^{\prime \prime} \Phi+B \Phi^{\prime \prime} \\
& \alpha_{0}=\frac{\omega^{2} \nu \Delta E}{c^{2}}-\frac{\Delta \epsilon \omega^{2} \xi_{-1} B \Phi}{c^{2}}-\frac{\Delta \epsilon \omega^{2} \xi_{+1} A \Phi}{c^{2}}+\left(\frac{\partial^{2}}{\partial x^{2}}+\frac{\partial^{2}}{\partial z^{2}}\right) \Delta E
\end{aligned}
$$


Now we assume $A(z)$ and $B(z)$ to be slowly varying functions of $z$ so that their second derivative can be neglected. Upon this assumption and because $\alpha_{-1}, \alpha_{0}, \alpha_{1}=0$ (plain from Eq.(5)), Eq.(6) yields:

$$
\begin{aligned}
\left(\frac{\partial^{2}}{\partial x^{2}}+\frac{\omega^{2} \nu}{c^{2}}-K^{2}+2 i K \frac{\partial}{\partial z}\right) A \Phi & =\frac{\Delta \epsilon \omega^{2}}{c^{2}}\left(\xi_{-1} \Delta E+\xi_{-2} B \Phi\right), \\
\left(\frac{\partial^{2}}{\partial x^{2}}+\frac{\omega^{2} \nu}{c^{2}}-K^{2}-2 i K \frac{\partial}{\partial z}\right) B \Phi & =\frac{\Delta \epsilon \omega^{2}}{c^{2}}\left(\xi_{+1} \Delta E+\xi_{+2} A \Phi\right), \\
\left(\frac{\partial^{2}}{\partial x^{2}}+\frac{\partial^{2}}{\partial z^{2}}+\frac{\omega^{2} \nu}{c^{2}}\right) \Delta E & =\frac{\Delta \epsilon \omega^{2}}{c^{2}}\left(\xi_{+1} A \Phi+\xi_{-1} B \Phi\right) .
\end{aligned}
$$

We assume, as well, that $\Delta E(x, z)$ in Eq.(7c) slowly varies with $z$, i.e., $\partial^{2} / \partial z^{2} \equiv 0$. Under this assumption, the solution of Eq.(7c) is given by:

$$
\Delta E(x, z)=\frac{\Delta \epsilon \omega^{2}}{c^{2}} \int\left[\xi_{+1}\left(x^{\prime}\right) A(z)+\xi_{-1}\left(x^{\prime}\right) B(z)\right] \Phi\left(x^{\prime}\right) G\left(x, x^{\prime}\right) \mathrm{d} x^{\prime},
$$

in which $G\left(x, x^{\prime}\right)$ is the Green's function that satisfies

$$
\left[\frac{\partial^{2}}{\partial x^{2}}+\nu \frac{\omega^{2}}{c^{2}} G\left(x, x^{\prime}\right)\right]=\delta\left(x-x^{\prime}\right)
$$

and have the same boundary conditions as $\Delta E(x, z)$. The construction of a suitable Green's function will be the object of Section 2.3.

We now deal with Eqs.(7a,7b). It has been emphasized above that $\Phi(x)$ is the transverse field of a given mode for the bare membrane. Accordingly $\Phi(x)$ must satisfy the wave equation

$$
\left.\frac{\partial^{2} \Phi(x)}{\partial x^{2}}+\left(\nu \frac{\omega_{0}^{2}}{c^{2}}-\beta^{2}\right)\right) \Phi(x)=0 .
$$

Furthermore, when perturbed by the periodic permittivity, the angular frequency $\omega_{0}$ of the homogeneous waveguide is shifted by a certain amount, say $\Delta \omega$, in such a way that the new (and complex) eigenfrequency reads:

$$
\omega=\omega_{0}+\Delta \omega
$$

If we introduce this last equation into the left-hand side of Eqs.(7a,7b), the first three terms grow into

$$
\frac{\partial^{2}}{\partial x^{2}}+\nu \frac{\omega_{0}^{2}}{c^{2}}-K^{2}+\nu \frac{\omega_{0} \Delta \omega}{c^{2}} .
$$

in which the second order angular frequency perturbation $\left(\Delta \omega^{2}\right)$ is neglected. Owing to Eq.(10) the sum of the first three terms in Eq.(12) vanishes; we can therefore reduce Eqs.(7a,7b) to

$$
\begin{aligned}
& \left(\nu \frac{\omega_{0} \Delta \omega}{c^{2}}+2 i K \frac{\partial}{\partial z}\right) A \Phi=\frac{\Delta \epsilon \omega^{2}}{c^{2}}\left(\xi_{-1} \Delta E+\xi_{-2} B\right) \Phi \\
& \left(\nu \frac{\omega_{0} \Delta \omega}{c^{2}}-2 i K \frac{\partial}{\partial z}\right) B \Phi=\frac{\Delta \epsilon \omega^{2}}{c^{2}}\left(\xi_{+1} \Delta E+\xi_{+2} A\right) \Phi .
\end{aligned}
$$

On multiplying this set of equations by $\Phi^{*}(x)$, integrating and assuming the following normalization condition for a given mode,

$$
\int_{-\infty}^{+\infty} \Phi(x) \Phi^{*}(x) \mathrm{d} x=1
$$


one obtains

$$
\begin{aligned}
& {\left[\frac{\omega_{0} \Delta \omega}{c^{2}} \int \nu \Phi \Phi^{*} \mathrm{~d} x+2 i K \frac{\partial}{\partial z}\right] A=\frac{\Delta \epsilon \omega^{2}}{c^{2}}\left[\int \Delta E \xi_{-1} \Phi^{*} \mathrm{~d} x+B \int \xi_{-2} \Phi \Phi^{*} \mathrm{~d} x\right]} \\
& {\left[\frac{\omega_{0} \Delta \omega}{c^{2}} \int \nu \Phi \Phi^{*} \mathrm{~d} x-2 i K \frac{\partial}{\partial z}\right] B=\frac{\Delta \epsilon \omega^{2}}{c^{2}}\left[\int \Delta E \xi_{+1} \Phi^{*} \mathrm{~d} x+A \int \xi_{+2} \Phi \Phi^{*} \mathrm{~d} x\right] .}
\end{aligned}
$$

Furthermore, the group velocity of a mode that propagates in a waveguide with propagation constant and frequency, $K$ and $\omega_{0}$ respectively, and with a prescribed field $\Phi(x)$ is given by (see Snyder ${ }^{6}$ for instance, Eq.(11$33)$ )

$$
v_{g}=\frac{c^{2} K}{\omega_{0}} \frac{\int \Phi(x) \Phi^{*}(x) \mathrm{d} x}{\int \nu(x) \Phi(x) \Phi^{*}(x) \mathrm{d} x},
$$

which means that the first term at the left-hand side of both equalities in Eq.(15) reduces to

$$
\frac{\omega_{0} \Delta \omega}{c^{2}} \int \nu(x) \Phi(x) \Phi^{*}(x) \mathrm{d} x=K \frac{\Delta \omega}{v_{g}}
$$

Upon introduction of the quantity $\Delta E(x, z)$ (given by Eq.(8)) into Eq.(15), the first term at the right-hand side of both equations read:

$$
\begin{aligned}
& \frac{\Delta \epsilon^{2} \omega^{4}}{c^{4}} \int_{x} \int_{x^{\prime}}\left[\xi_{+1}\left(x^{\prime}\right) A(z)+\xi_{-1}\left(x^{\prime}\right) B(z)\right] \xi_{-1}(x) \Phi\left(x^{\prime}\right) \Phi^{*}(x) G\left(x, x^{\prime}\right) \mathrm{d} x \mathrm{~d} x^{\prime}, \\
& \frac{\Delta \epsilon^{2} \omega^{4}}{c^{4}} \int_{x} \int_{x^{\prime}}\left[\xi_{+1}\left(x^{\prime}\right) A(z)+\xi_{-1}\left(x^{\prime}\right) B(z)\right] \xi_{+1}(x) \Phi\left(x^{\prime}\right) \Phi^{*}(x) G\left(x, x^{\prime}\right) \mathrm{d} x \mathrm{~d} x^{\prime} .
\end{aligned}
$$

Furthermore, since the grating is symmetric about $z\left(\epsilon_{p c m}(x, z)=\epsilon_{p c m}(x,-z)\right), \xi_{m}$ and $\xi_{-m}$ must be equal according to Eq.(1); thus the two equalities in Eq.(18) turn out to take the same form:

$$
[A(z)+B(z)] \frac{\Delta \epsilon^{2} \omega^{4}}{c^{4}} \int_{x} \int_{x^{\prime}} \xi_{+1}\left(x^{\prime}\right) \xi_{+1}(x) \Phi\left(x^{\prime}\right) \Phi^{*}(x) G\left(x, x^{\prime}\right) \mathrm{d} x \mathrm{~d} x^{\prime} .
$$

Finally, if one defines two coefficients,

$$
\begin{aligned}
& h_{1}=\frac{i \Delta \epsilon^{2} \omega^{4}}{2 K c^{4}} \int_{0}^{d} \int_{0}^{d} G\left(x, x^{\prime}\right) \xi_{1}\left(x^{\prime}\right) \xi_{1}(x) \Phi\left(x^{\prime}\right) \Phi(x)^{*} \mathrm{~d} x \mathrm{~d} x^{\prime}, \\
& h_{2}=-\frac{\Delta \epsilon \omega^{2}}{2 K c^{2}} \int_{0}^{d} \xi_{+2}(x) \Phi(x) \Phi^{*}(x) \mathrm{d} x,
\end{aligned}
$$

Eq.(15), together with Eq.(19), becomes

$$
\begin{aligned}
& {\left[\frac{\Delta \omega}{2 v_{g}}+i \frac{\partial}{\partial z}\right] A(z)+h_{2} B(z)+i h_{1}[A(z)+B(z)]=0} \\
& {\left[\frac{\Delta \omega}{2 v_{g}}-i \frac{\partial}{\partial z}\right] B(z)+h_{2} A(z)+i h_{1}[A(z)+B(z)]=0 .}
\end{aligned}
$$

The last set of equations are the one first derived by $\mathrm{KH}$ in [4] to study DFB lasers with second-order gratings (note however that $\Delta \omega / v_{g}$ is here multiplied by $1 / 2$ compared to KH's formula).

\subsection{Dispersion relation and radiation losses for PCMs from the $h_{1}$ and $h_{2}$ coefficients}

In this paper the KH's approach is pushed further in order to describe the radiation losses in PCMs. The parameters $h_{1}$ and $h_{2}$ are shown to define a dispersion relation which provides insight into the radiation losses experienced by the PCM. 
Assuming $A(z) \propto B(z) \propto e^{i k_{z} z}$ the resolution of Eq.(21) leads to an eigenvalue equation whose solution is

$$
\frac{\Delta \omega}{2 v_{g}}=-i h 1 \pm \sqrt{\left(i h_{1}+h_{2}\right)^{2}+k_{z}^{2}}
$$

Since we are considering a normal incidence $k_{z}=0$ implies that

$$
\frac{\Delta \omega}{2 v_{g}}= \begin{cases}+h_{2} & \text { (plus sign) } \\ -\left(h_{2}+2 i h_{1}\right) & \text { (minus sign) } .\end{cases}
$$

We start the analysis by noticing that we have a measure of the deviation experienced by $\omega_{0}$ at the $\Gamma$ point of the membrane dispersion diagram when the perturbation is accounted for. Depending on the plus or minus sign, two sets of dispersion results are obtained: (i) If the minus sign is considered, the PCM's eigenfrequency is deviated from that of the bare waveguide by a magnitude $2 i h_{1}+h_{2}$. With reference to Eq.(20), we notice that $h_{1}$ is complex while $h_{2}$ is real. As a result the deviation is in this case a complex number. The real part of it accounts for the opening of a bandgap and the imaginary part $\left(2 \operatorname{Re}\left(h_{1}\right)\right)$ describes the radiation losses. (ii) The plus sign yields a real deviation of the frequency, viz., the opening of a bandgap with no radiation losses. Resonances, lying into the light cone, with infinite lifetime at discrete k points on certain bands were intensively treated in the literature; see, e.g., [7].

To sum up, we know that corrugating a waveguide is of twofold impact. First, the alteration of the horizontal propagation of light in the structure induces a bandgap in the dispersion diagram. Second, in case of operating above the light line, the corrugation provokes the vertical coupling of the radiation and guided modes in the PCM. It is interesting to note that the coefficients $h_{1}$ and $h_{2}$ can be apprehended as the mathematical counterpart of this twofold impact.

\subsection{Green's Function}

We have seen that the radiation losses are contained into the parameter $h_{1}$ given in Eq.(20a). This expression involves a Green's function. KH's suggestion for the Green's function did not explicitly consider the surrounding medium, which results in disregarding the multiple reflections that take place at the interfaces of the structure. An intent proposed by Rosenblatt et al. did take into account the effect of multiple reflections but they finally obtained an expression for the radiation losses that is not invariant by permutation of the reflectivities at the upper and lower boundaries (see Eq.(C2) in [8]). It is therefore essential to construct a suitable Green's function; such a task results in the purpose of this section.

We recall first that the Green's Function of a differential equation such as Eq.(7c) is constructed from two independent and non-trivial solutions of the homogeneous form of it, each satisfying the boundary conditions. In the case of the PCM with thickness $d$, if

1. $\Phi^{+}(x)$ is a solution of Eq.(7c) in the range $x^{\prime}<x<d$ which satisfies the boundary conditions at $x=d$, and

2. $\Phi^{-}(x)$ is a solution of Eq.(7c) in the range $0<x<x^{\prime}$ which satisfies the boundary conditions at $x=0$

then the Green's function can be expressed as

$$
G\left(x, x^{\prime}\right)= \begin{cases}\frac{\Phi^{-}\left(x^{\prime}\right)}{W\left(x^{\prime}\right)} \Phi^{+}(x) & \text { if } x \geq x^{\prime}, \\ \frac{\Phi^{+}\left(x^{\prime}\right)}{W\left(x^{\prime}\right)} \Phi^{-}(x) & \text { if } x \leq x^{\prime},\end{cases}
$$

where $W\left(x^{\prime}\right)$ is the Wronskian of $\Phi^{+}$and $\Phi^{-}$at $x=x^{\prime}$

$$
W\left(x^{\prime}\right)=\left.\Phi^{-}\left(x^{\prime}\right) \frac{\partial \Phi^{+}(x)}{\partial x}\right|_{x=x^{\prime}}-\left.\Phi^{+}\left(x^{\prime}\right) \frac{\partial \Phi^{-}(x)}{\partial x}\right|_{x=x^{\prime}} .
$$


For illustration's sake, KH chose

$$
\begin{aligned}
& \Phi^{+}(x)=\exp \left(+i k_{x} x\right), \\
& \Phi^{-}(x)=\exp \left(-i k_{x} x\right),
\end{aligned}
$$

leading to

$$
W\left(x^{\prime}\right)=2 i k_{x}
$$

and

$$
G\left(x, x^{\prime}\right)= \begin{cases}\frac{\exp \left[+i k_{x}\left(x-x^{\prime}\right)\right]}{2 i k_{x}} & \text { if } x \geq x^{\prime}, \\ \frac{\exp \left[-i k_{x}\left(x-x^{\prime}\right)\right]}{2 i k_{x}} & \text { if } x \leq x^{\prime} .\end{cases}
$$

However, the above $\Phi^{+}$and $\Phi^{+}$functions are two bare plane waves unable to provide accounts of multiple reflections at the interfaces of the membrane. We shall construct a Green's function that figures out such a limitation. As depicted in Fig.1, our suggestion is to choose $\Phi^{+}$to be the field inside a sheet - with wave number $k_{2}$ and surrounded by two semi-infinite media $k_{1}$ and $k_{3}$ - when a wave is incoming from medium 1. Oppositely, the function $\Phi^{-}$is chosen such that the incident wave is incoming from medium 3.

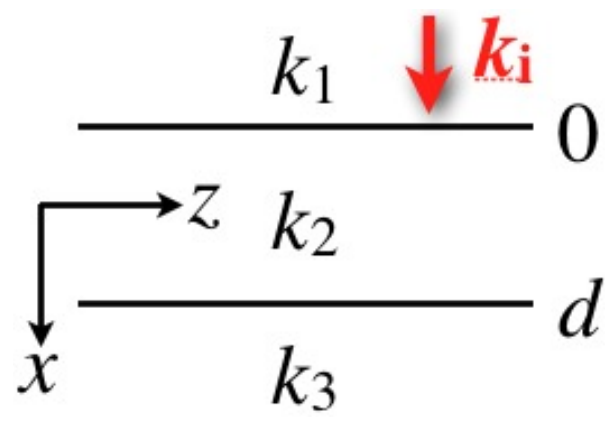

(a)

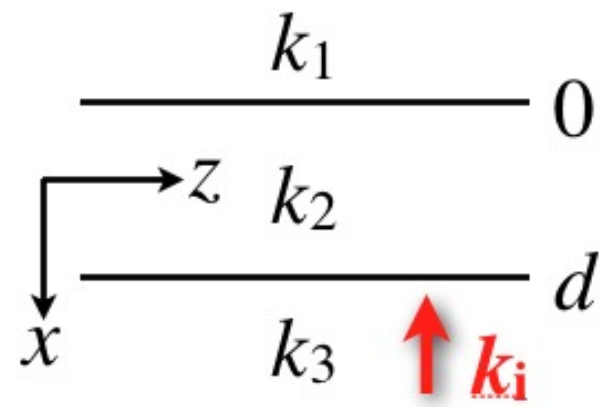

(b)

Figure 1. (a) Wave incident from medium 1 for the construction of $\Phi_{+}$. (b) Wave incident from medium 3 for the construction of $\Phi_{-}$.

Regarding the derivation of $\Phi^{+}$, we have an academic 3 layers problem that is solved by expressing the electric field

$$
\begin{aligned}
& E_{i}(x)=E_{0} \mathrm{e}^{i k_{1} x-i \omega t}, \\
& E_{r}(x)=E_{1} \mathrm{e}^{-i k_{1} x-i \omega t}, \\
& E_{2}(x)=\left[E_{2}^{+} \mathrm{e}^{i k_{2} x}+E_{2}^{-} \mathrm{e}^{-i k_{2} x}\right] \mathrm{e}^{-i \omega t}, \\
& E_{t}(x)=E_{3} \mathrm{e}^{i k_{3} x-i \omega t} .
\end{aligned}
$$

in the three media, equations from which the magnetic field $\mathbf{H}=\frac{1}{\mu \mu_{0} \omega} \mathbf{k} \times \mathbf{E}$ can be derived:

$$
\begin{aligned}
& H_{i}(x)=\frac{k_{1}}{\omega \mu_{1} \mu_{0}} E_{0} \mathrm{e}^{i k_{1} x-i \omega t}, \\
& H_{r}(x)=-\frac{k_{1}}{\omega \mu_{1} \mu_{0}} E_{1} \mathrm{e}^{-i k_{1} x-i \omega t}, \\
& H_{2}(x)=\frac{k_{2}}{\omega \mu_{2} \mu_{0}}\left[E_{2}^{+} \mathrm{e}^{i k_{2} x}-E_{2}^{-} \mathrm{e}^{-i k_{2} x}\right] \mathrm{e}^{-i \omega t}, \\
& H_{t}(x)=\frac{k_{2}}{\omega \mu_{3} \mu_{0}} E_{3} \mathrm{e}^{i k_{3} x-i \omega t} .
\end{aligned}
$$


By employing the continuity of the tangent fields at both interface, the usual expressions

$$
\begin{aligned}
& E_{1}=E_{0} \frac{\mathrm{e}^{2 i h k_{2}}\left(k_{1}+k_{2}\right)\left(k_{2}-k_{3}\right)+\left(k_{1}-k_{2}\right)\left(k_{2}+k_{3}\right)}{\mathrm{e}^{2 i h k_{2}}\left(k_{1}-k_{2}\right)\left(k_{2}-k_{3}\right)+\left(k_{1}+k_{2}\right)\left(k_{2}+k_{3}\right)}, \\
& E_{2}^{+}=E_{0} \frac{2 k_{1}\left(k_{2}+k_{3}\right)}{\mathrm{e}^{2 i h k_{2}}\left(k_{1}-k_{2}\right)\left(k_{2}-k_{3}\right)+\left(k_{1}+k_{2}\right)\left(k_{2}+k_{3}\right)}, \\
& E_{2}^{-}=E_{0} \frac{2 \mathrm{e}^{2 i h k_{2}} k_{1}\left(k_{2}-k_{3}\right)}{\mathrm{e}^{2 i h k_{2}}\left(k_{1}-k_{2}\right)\left(k_{2}-k_{3}\right)+\left(k_{1}+k_{2}\right)\left(k_{2}+k_{3}\right)}, \\
& E_{3}=-E_{0} \frac{4 \mathrm{e}^{i h\left(k_{2}-k_{3}\right)} k_{1} k_{2}}{\mathrm{e}^{2 i h k_{2}}\left(-k_{1}+k_{2}\right)\left(k_{2}-k_{3}\right)-\left(k_{1}+k_{2}\right)\left(k_{2}+k_{3}\right)},
\end{aligned}
$$

are obtained. From Fresnel's relations (expressing the intrinsic reflection and transmission coefficients at the interfaces) we can express $k_{1}$ and $k_{3}$ against $k_{2}, \rho_{21}$, and $\rho_{23}$

$$
\begin{aligned}
& \rho_{21}=\frac{k_{2}-k_{1}}{k_{2}+k_{1}}, \Rightarrow \begin{aligned}
k_{1} & =k_{2} \frac{1-\rho_{21}}{1+\rho_{21}}, \\
k_{3} & =k_{2} \frac{1-\rho_{23}}{1+\rho_{23}},
\end{aligned} \\
& \rho_{23}=\frac{k_{2}-k_{3}}{k_{2}+k_{3}}, \Rightarrow k_{3}=k_{2} \frac{1-\rho_{23}}{1+\rho_{23}},
\end{aligned}
$$

which allows to rewrite Eq.(31) as

$$
\begin{aligned}
& E_{1}=E_{0} \frac{\rho_{21}-\rho_{23} \mathrm{e}^{2 i h k_{2}}}{-1+\rho_{21} \rho_{23} \mathrm{e}^{2 i h k_{2}}}, \\
& E_{2}^{+}=E_{0} \frac{-1+\rho_{21}}{-1+\rho_{21} \rho_{23} \mathrm{e}^{2 i h k_{2}}}, \\
& E_{2}^{-}=E_{0} \mathrm{e}^{2 i h k_{2}} \frac{\left(-1+\rho_{21}\right) \rho_{23}}{-1+\rho_{21} \rho_{23} \mathrm{e}^{2 i h k_{2}}}, \\
& E_{3}=E_{0} \mathrm{e}^{\frac{2 i h k_{2} \rho_{23}}{1+\rho_{23}}} \frac{\left(-1+\rho_{21}\right)\left(1+\rho_{23}\right)}{-1+\rho_{21} \rho_{23} \mathrm{e}^{2 i h k_{2}}} .
\end{aligned}
$$

As mentioned above, we suggest that $\Phi^{+}$be $E_{2}$, i.e.,

$$
\Phi^{+}(x)=E_{0}\left[\frac{-1+\rho_{21}}{-1+\rho_{21} \rho_{23} \mathrm{e}^{2 i h k_{2}}} \exp \left(i k_{2} x\right)+\mathrm{e}^{2 i h k_{2}} \frac{\left(-1+\rho_{21}\right) \rho_{23}}{-1+\rho_{21} \rho_{23} \mathrm{e}^{2 i h k_{2}}} \exp \left(-i k_{2} x\right)\right] .
$$

The construction of $\Phi_{-}$employs the same process. We start by expressing the field incoming from medium 3

$$
\begin{aligned}
& E_{i}(x)=E_{0} \mathrm{e}^{-i k_{3} x-i \omega t}, \\
& E_{r}(x)=E_{3} \mathrm{e}^{+i k_{3} x-i \omega t}, \\
& E_{2}(x)=\left[E_{2}^{+} \mathrm{e}^{i k_{2} x}+E_{2}^{-} \mathrm{e}^{-i k_{2} x}\right] \mathrm{e}^{-i \omega t}, \\
& E_{t}(x)=E_{1} \mathrm{e}^{-i k_{1} x-i \omega t},
\end{aligned}
$$

to eventually get:

$$
\Phi^{-}(x)=E_{0} \mathrm{e}^{\frac{2 i h k_{2} \rho_{23}}{1+\rho_{23}}}\left[\frac{\left(-1+\rho_{23}\right) \rho_{21}}{-1+\rho_{21} \rho_{23} \mathrm{e}^{2 i h k_{2}}} \exp \left(i k_{2} x\right)+\frac{-1+\rho_{23}}{-1+\rho_{21} \rho_{23} \mathrm{e}^{2 i h k_{2}}} \exp \left(-i k_{2} x\right)\right] .
$$

The introduction of Eqs.(34,36) into Eq.(24) finally provides the Green's function:

$$
G\left(x, x^{\prime}\right)=\frac{-i}{2 k_{2}\left(\rho_{21} \rho_{23} \mathrm{e}^{2 i k_{2} h}-1\right)}\left\{\begin{array}{cc}
\mathrm{e}^{i k_{2}\left(x-x^{\prime}\right)}+\rho_{21} \rho_{23} \mathrm{e}^{2 i k_{2} h} \mathrm{e}^{-i k_{2}\left(x-x^{\prime}\right)}+ & \text { if } x \geq x^{\prime}, \\
\rho_{21} \mathrm{e}^{i k_{2}\left(x+x^{\prime}\right)}+\rho_{23} \mathrm{e}^{2 i k_{2} h} \mathrm{e}^{-i k_{2}\left(x+x^{\prime}\right)} & \\
\mathrm{e}^{-i k_{2}\left(x-x^{\prime}\right)}+\rho_{21} \rho_{23} \mathrm{e}^{2 i k_{2} h} \mathrm{e}^{i k_{2}\left(x-x^{\prime}\right)}+ & \text { if } x \leq x^{\prime} . \\
\rho_{21} \mathrm{e}^{i k_{2}\left(x+x^{\prime}\right)}+\rho_{23} \mathrm{e}^{2 i k_{2} h} \mathrm{e}^{-i k_{2}\left(x+x^{\prime}\right)} &
\end{array}\right.
$$




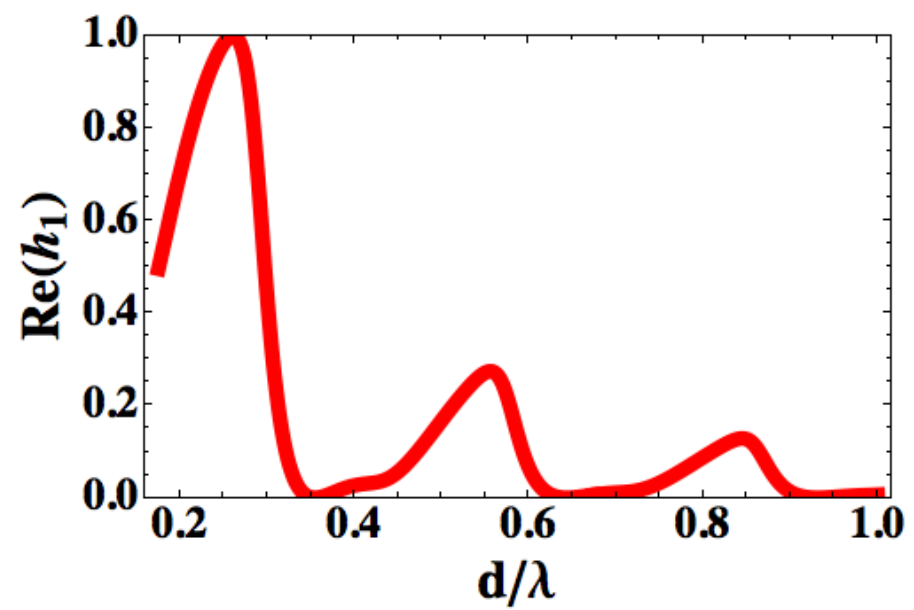

Figure 2. Radiation losses in terms of the ratio $d / \lambda$ of a silicon membrane embedded in air (we have assumed that $\left.n_{\mathrm{Si}}=3.481\right)$. The curve has been normalized to the maximum value of $\Re\left(h_{1}\right)$.

\section{DISCUSSION}

\subsection{Calculation of the radiation losses for a symmetric mode}

The intricate form of both $h_{1}$ and $G\left(x, x^{\prime}\right)$ makes it difficult to evaluate the radiation losses. However, it has been shown in [5] that $h_{1}$ can be analytically calculated if a symmetric mode,

$$
\Phi(x)=\cos \kappa\left(x-\frac{d}{2}\right),
$$

is assumed. The quantity $\kappa$ is the vertical component of the wavevector in the unpatterned waveguide. By using $\mathrm{G}\left(\mathrm{x}, \mathrm{x}^{\prime}\right)$ given in Eq.(37), a pretty tough calculation gives

$$
\begin{aligned}
& \operatorname{Re}\left(h_{1}\right)=\frac{\Delta \epsilon^{2} \omega^{4} \xi_{1}^{2}}{4 k_{2} K c^{4}} \\
& \times \frac{\left[\rho_{21} \rho_{23}-1\right]\left[1+\rho_{21} \rho_{23}+\left(\rho_{21}+\rho_{23}\right) \cos d k_{2}\right]}{1+\rho_{21}^{2} \rho_{23}^{2}-2 \rho_{21} \rho_{23} \cos 2 d k_{2}} \\
& \times\left[\frac{\sin \frac{d}{2}\left(k_{2}-\kappa\right)}{k_{2}-\kappa}+\frac{\sin \frac{d}{2}\left(k_{2}+\kappa\right)}{k_{2}+\kappa}\right]^{2} .
\end{aligned}
$$

A lot has been said in [5] about the information that can be drawn from Eq.(39). We recall here the main features:

1) We clearly see that the expression for $h_{1}$ splits into two main terms - we leave out of the analysis the pre-factor $\left(\Delta \epsilon^{2} \omega^{4} \xi_{1}^{2}\right) /\left(4 k_{2} K c^{4}\right)$. There is an interferential term that accounts for the surrounding medium (it depends on the reflectivities $\rho_{21}$ and $\rho_{23}$ ) and that, interestingly, turns out to be identical to what one may obtain by a Couple Mode Theory treatment of the problem. Note in passing that this term vanishes if one would use the Green's function derived by KH (Eq.(28)). The second term is the clear signature of the overlap of radiation and guided modes along the membrane thickness inasmuch as

$$
\frac{\sin \frac{d}{2}\left(k_{2}-\kappa\right)}{k_{2}-\kappa}+\frac{\sin \frac{d}{2}\left(k_{2}+\kappa\right)}{k_{2}+\kappa}=\left|\int_{0}^{d} \mathrm{e}^{i k_{2} x} \Phi(x) \mathrm{d} x\right| .
$$

A usual statement is to consider a resonance in a PCM as the result of the coupling between radiation and guided modes. ${ }^{9}$ Through our perturbative model, we give an analytical justification to this perception by showing that 


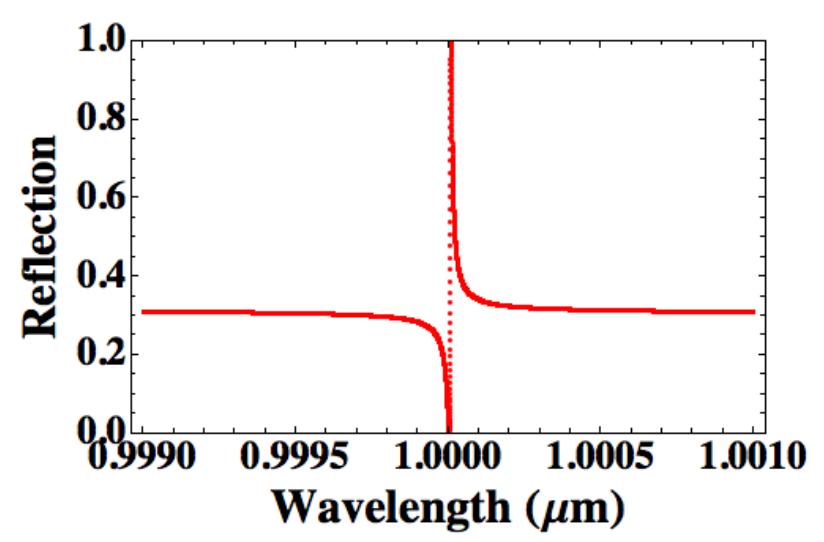

(a)

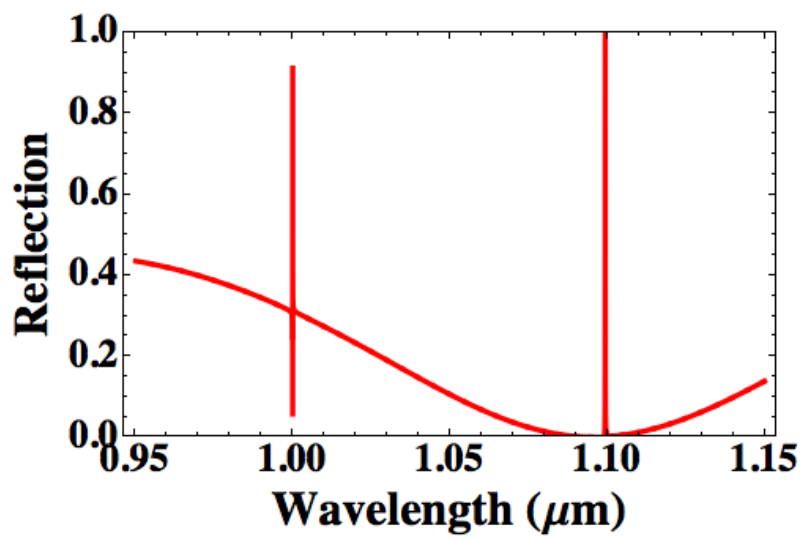

(b)

Figure 3. (a) Reflectivity in terms of the wavelength if $d=0.3483 \mu \mathrm{m}$. A strong Fano's resonance is observed at $\lambda=1 \mu \mathrm{m}$ for this particular thickness. (b) Reflectivity in terms of the wavelength if the angle of incidence is tilted by $1^{\circ}$. A dark mode is revealed around $\lambda=1.1 \mu \mathrm{m}$.

the $\mathrm{Q}$ factor is proportional to the vertical overlap integral between the electromagnetic field distributions of these eigenmodes.

2) Depicted in Fig.2 is the plot of $h_{1}$ against the ratio $d / \lambda ; \kappa$ being obtained by means of the calculation of the eigenvalues of the modes in a bare waveguide for TE polarization $\left(\mathbf{E}=E_{y} \hat{\mathbf{y}}\right)$. The curve is an oscillating function that exhibits true zero values. This is an important result given that it demonstrates the possibility of reaching infinite $\mathrm{Q}$ factors. What is more, the only parameter that controls such a feature is the thickness of the membrane. In addition, we showed that the thicknesses leading to infinite Q factors are universal since they turns out to be largely independent of the PCM constitutive materials as long as the index contrast between the membrane and the environing medium is high enough. ${ }^{5}$

\subsection{Vertical versus horizontal coupling}

Let us consider a PCM (made up of an alternateness of Si-Air) whose constitutive parameters are $d=0.3483 \mu \mathrm{m}$, $\Lambda=0.59264 \mu \mathrm{m}$, and fill factor $F=0.5$. This value of $d=0.3483 \mu \mathrm{m}$ has been chosen so that $h_{1} \simeq 0$ and, accordingly, a sharp Fano's resonance arises at $\lambda=1 \mu \mathrm{m}$ as it can be seen in Fig.3(a). Note that $F$ plays no role here, the value of $50 \%$ was arbitrary chosen and the result would be the same if another $F$ would be elected. ${ }^{10}$ In Fig.4(a) is mapped the corresponding electric field.

The incident wave is now tilted by $1^{\circ}$. The consequence of this slight variation on the angle is the appearance of a narrow resonance near $\lambda=1 \mu \mathrm{m}$. We therefore understand that at $\Gamma$ we had a eigenmode with infinite $\mathrm{Q}$ factor, which starts decaying into the continuum when the angle is tilted. One may foresee the influence of the symmetry on this phenomenon. This is confirmed by the mapping of the field configuration that is represented in Fig.4(b). The field shows a clear antisymmetry about $z=0$, as a result its integral along a period vanishes. In this case it is the horizontal arrangement of the crystal that authorizes no coupling to outgoing waves. Such a dark mode is predicted by our perturbative approach through the branch of the dispersion relation in Eq.(23) that remains real.

Oppositely, the branch that is complex comprises radiative losses by means of $h_{1}$ and it turns out that this parameter vanishes for well determined values of the membrane thickness. However, the examination of Fig.4(a) provides no direct clue regarding the mechanism leading to the high-Q regime. We do realize that the energy is well confined along the membrane thickness but there is no vanishing integral in this case, for the field is not a separable function of $x$ and $z$ (as in Fig.4(b)). We suspect that the explanation there must be complex interferences at the upper and lower interface of the membrane; this is reminiscent of the approach developed in [11]. 


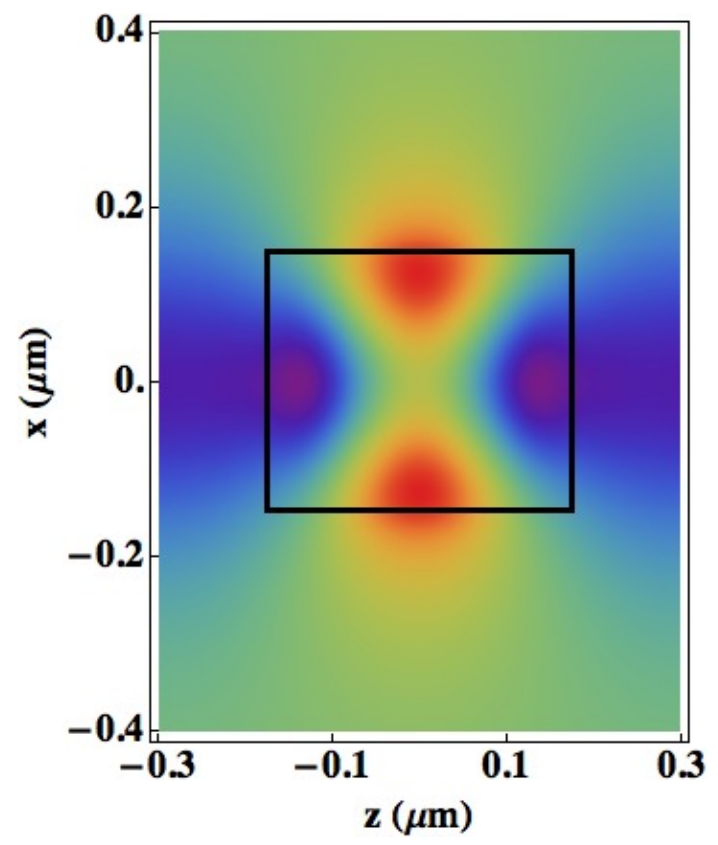

(a)

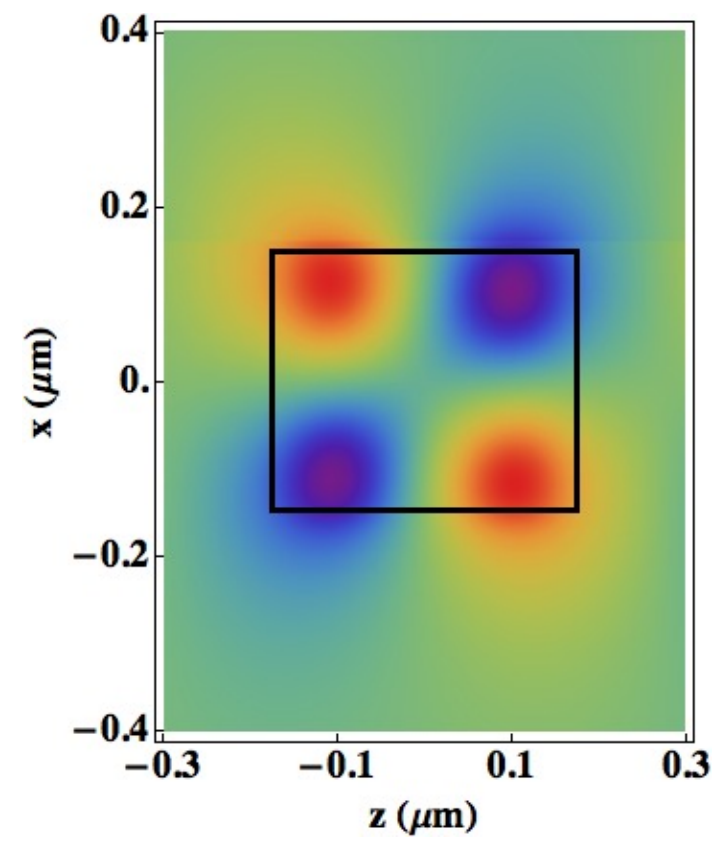

(b)

Figure 4. Mapping of the $E$-field for (a) the Fano's resonance at $\lambda=1 \mu \mathrm{m}$ and (b) the dark mode at $\lambda=1.1 \mu \mathrm{m}$.

\section{CONCLUSION}

The equations sustaining a perturbative approach able to describe the radiation losses for a high contrast grating are presented with great details. The model provides insight on the behavior of such structures, e.g. their ability of supporting resonances endowed with an infinite Q factor. In this last mechanism the optical thickness of the membrane is the key parameter irrespective of the resonator itself and even of the environment as long as the index contrast is enough. Furthermore, from a phenomenological perspective, the radiation losses are shown to be proportional to the vertical overlap integral between the guided and radiative modes.

Overall, the developments presented here constitute a useful tool to understand and engineer the spectral characteristic of nanophotonic systems, a feature that can in particular be exploited in the realm of energy efficiency.

\section{ACKNOWLEDGMENTS}

This work was funded by the French Agence Nationale de la Recherche (ANR), IDEE project. The authors acknowledge Laurent Carrel for helpful support regarding INL computing facilities. Cédric Blanchard expresses gratitude to John Elkhoury for support in the redaction of the manuscript.

\section{REFERENCES}

1. E. Rephaeli, A. Raman, and S. Fan, "Ultrabroadband photonic structures to achieve high-performance daytime radiative cooling," Nano Letters 13(4), pp. 1457-1461, 2013.

2. A. P. Raman, M. A. Anoma, L. Zhu, E. Rephaeli, and S. Fan, "Passive radiative cooling below ambient air temperature under direct sunlight," Nature 515(7528), pp. 540-544, 2014.

3. J. A. Lenert, D. M. Bierman, Y. Nam, W. R. Chan, I. Celanović, M. Soljačić, and E. N. Wang, "A nanophotonic solar thermophotovoltaic device," Nat. Nano. 9, pp. 126-130, 2014.

4. R. F. Kazarinov and C. H. Henry, "Second-order distributed feedback lasers with mode selection provided by first-order radiation losses," IEEE J. Quant. Electron. QE-21(2), pp. 144-150, 1985. 
5. C. Blanchard, P. Viktorovitch, and X. Letartre, "Perturbation approach for the control of the quality factor in photonic crystal membranes: application to selective absorbers," Phys. Rev. A 90(3), p. 033824, 2014.

6. A. W. Snyder and J. D. Love, Optical waveguide theory, Chapman and Hall, London and New-York, 1983.

7. C. W. Hsu, B. Zhen, J. Lee, S.-L. Chua, S. G. Johnson, J. D. Joannopoulos, and M. Soljačić, "Observation of trapped light within the radiation continuum," Nature 499(7457), pp. 188-191, 2013.

8. D. Rosenblatt, A. Sharon, and A. A. Friesem, "Resonant grating waveguide structures," IEEE J. Quant. Electron. 33(11), pp. 2038-2059, 1997.

9. R. Magnusson and S. S. Wang, "New principle for optical filters," Appl. Phys. Lett. 61(9), pp. 1022-1024, 1992.

10. X. Letartre, C. Blanchard, R. Peretti, and C. Sciancalepore, "Confinement of photons and control of their emission using surface addressable photonic crystal membrane," in SPIE Photonics West 2014, 1-6 Feb. 2014.

11. V. Karagodsky and C. J. Chang-Hasnain, "Physics of near-wavelength high contrast gratings," Opt. Express 20(10), pp. 10888-10895, 2012. 